\title{
Nanoparticle ligand exchange and its effects at the nanoparticle-cell membrane interface
}

Xinyi Wang, Xiaofeng Wang, Xuan Bai, Liang Yan, Tao Liu, Mingzhe Wang, Youtao Song, Guoqing Hu, Zhanjun Gu, Qing Robert Miao, and Chunying Chen

Nano Lett., Just Accepted Manuscript • DOI: 10.1021/acs.nanolett.8b02638 • Publication Date (Web): 15 Oct 2018

Downloaded from http://pubs.acs.org on October 16, 2018

\section{Just Accepted}

"Just Accepted" manuscripts have been peer-reviewed and accepted for publication. They are posted online prior to technical editing, formatting for publication and author proofing. The American Chemical Society provides "Just Accepted" as a service to the research community to expedite the dissemination of scientific material as soon as possible after acceptance. "Just Accepted" manuscripts appear in full in PDF format accompanied by an HTML abstract. "Just Accepted" manuscripts have been fully peer reviewed, but should not be considered the official version of record. They are citable by the Digital Object Identifier (DOI®). "Just Accepted" is an optional service offered to authors. Therefore, the "Just Accepted" Web site may not include all articles that will be published in the journal. After a manuscript is technically edited and formatted, it will be removed from the "Just Accepted" Web site and published as an ASAP article. Note that technical editing may introduce minor changes to the manuscript text and/or graphics which could affect content, and all legal disclaimers and ethical guidelines that apply to the journal pertain. ACS cannot be held responsible for errors or consequences arising from the use of information contained in these "Just Accepted" manuscripts. 


\title{
Nanoparticle ligand exchange and its effects at the nanoparticle-cell membrane interface
}

Xinyi Wang, ${ }^{\dagger, \dagger},{ }^{\dagger}$ Xiaofeng Wang, ${ }^{\|}$Xuan Bai, ${ }^{\perp}$ Liang Yan,, Tao Liu,${ }^{\dagger}$ Mingzhe Wang, ${ }^{\dagger}$ Youtao

Song, ${ }^{\S}$ Guoqing Hu, ${ }^{\perp}$ Zhanjun Gu,,${ }^{\|}$Qing Miao, ${ }^{\dagger}$ Chunying Chen ${ }^{*}{ }^{\dagger}$

${ }^{\dagger}$ CAS Key Laboratory for Biomedical Effects of Nanomaterials and Nanosafety, \&CAS Center for Excellence in Nanoscience, National Center for Nanoscienceand Technology of China, and University of Chinese Academy of Sciences, Beijing 100190, China;

$\ddagger$ College of Sciences, Shenyang Agricultural University, Shenyang 110161, China;

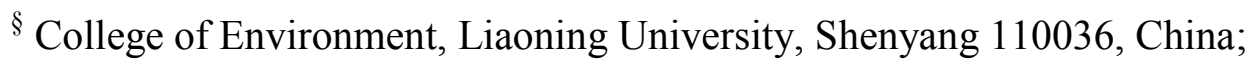

" CAS Key Laboratory for Biomedical Effects of Nanomaterials and Nanosafety, Institute of High Energy Physics, Chinese Academy of Sciences (CAS), and University of Chinese Academy of Sciences, Beijing 100049, China;

${ }^{\perp}$ The State Key Laboratory of Nonlinear Mechanics (LNM), Institute of Mechanics, Chinese Academy of Sciences, and University of Chinese Academy of Sciences, Beijing 100190, China

\author{
*Correspondence author: Prof. Chunying Chen \\ National Center for Nanoscience and Technology, Chinese Academy of Science \\ No.11 Beiyitiao, Zhongguancun, Beijing 100190, China \\ Email: chenchy@nanoctr.cn \\ Phone: +86108254 5560, Fax: +861062656765
}




\begin{abstract}
The nanoparticle (nano)-cell membrane interface is one of the most important interactions determining the fate of nanoparticles (NPs), which can stimulate a series of biological events, allowing theranostic and other biomedical applications. So far, there remains a lack of knowledge about the mechanisms governing the nano-cell membrane interface, especially the impact of ligand exchange, in which molecules on the nano-surface become replaced with components of the cell membrane, resulting in unique interfacial phenomena. Herein, we describe a family of gold nanoparticles (AuNPs) of the same core size ( 13 nm core), modified with 12 different kinds of surface ligands, and the effects of their exchangeable ligands on both nanosupported lipid bilayers (SLBs) and nano-natural cell membrane interfaces. The ligands are categorized according to their molecular weight, charge and bonding modes (physisorption or chemisorption). Importantly, we found that, depending on the adsorption affinity and size of ligand molecules, physisorbed ligands on the surface of NPs can be exchanged with lipid molecules. At a ligand exchange-dominated interface, the AuNPs typically aggregated into an ordered monolayer in the lipid bilayers, subsequently affecting cell membrane integrity, NP uptake efficiency and the NP endocytosis pathways. These findings advance our understanding of the underlying mechanisms of the biological effects of nanoparticles from a new point of view and will aid in the design of novel, safe and effective nanomaterials for biomedicine.
\end{abstract}

KEYWORDS: Nanoparticles, surface ligand exchange, nano-cell membrane interface, nanobiological effects, molecular dynamics simulation 


\section{INTRODUCTION}

A recent dramatic increase in the use of nanomaterials in biology and medicine has raised the issue of the potential toxicity of these formulations. ${ }^{1,2}$ Interactions between nanomaterials and biological systems can be essentially seen as a series of nano-bio interface events occurring at different size magnitudes, involving biomacromolecules, membranes, organelles, cells etc.. ${ }^{3,4}$ The cell membrane, as the primary defensive barrier of the cell, plays a key role in the uptake of nanoparticles (NPs) and the subsequent biocompatible or adverse outcomes. ${ }^{5-7}$ NPs can adhere to the amphiphilic lipid bilayer surface or become inserted into the bilayer alkane chain region, via hydrophilic/hydrophobic interactions, where they can disturb phospholipid bilayers and block membrane proteins. ${ }^{8,9}$ Therefore, investigating the underlying mechanisms of the nano-cell membrane interface is a key entry point for understanding nanosafety issues.

In spite of much research effort, understanding the nano-cell membrane interface remains challenging due to the diversity of NPs with unique physicochemical parameters and the dynamic characteristics of the interfacial interactions. ${ }^{10,11}$ The intrinsic characteristics of nanomaterials, such as size, shape, roughness, and surface chemistry, are usually important factors to be considered in the study of nano-bio interfaces. ${ }^{12,13}$ However, these intrinsic nanomaterial properties are often shielded or altered by ligands on the nanomaterial surface, which are generally applied to decrease the high surface energy of NPs, stabilize the nano colloid system, and/or functionalize the NPs. ${ }^{14,15}$ Thus, the actual interactions between NPs and biological systems are mediated by what has been unintentionally adsorbed or intentionally layered onto the surface of the NPs, rather than the unaltered surface of nascent NPs. ${ }^{16,17}$ There are many unintentional sources of ligands, such as chemical moieties adopted from the nanomaterial preparation ${ }^{15}$ and biomolecules adsorbed from the biological microenvironment, forming a corona. ${ }^{18,19}$ Therefore, a typical nano- 
cell membrane interface includes the NPs, surface ligand moieties, and the cell membrane elements composed of biomolecules and patchy structures. ${ }^{3}$ Thus, ligands in the midst of the contact area at the nano-cell membrane interface play a vital role in interfacial interactions.

In most cases, the specific properties of ligands are major contributors to the biological effects or toxicity induced by nanomaterials. However, dynamic ligand exchange on the nano-surface is a potential factor that is often ignored. Chemically, ligands adhere to the nano-surface mainly through 'chemisorption' and 'physisorption' pathways. Physisorbed ligands are easily replaced by cell membrane components, such as proteins and phospholipid species, when the NPs approach the cell membrane, as driven by thermodynamics and kinetics. ${ }^{20,21}$ Ligand exchange is a very complicated occurrence, involving multiple interaction potentials/forces. ${ }^{22-24}$ In the ligandexchange process, the intrinsic characteristics of the NP core (nanocore) are exposed and subsequent interactions of varying types ensue. These interactions, including ligand-ligand, ligandparticle, particle-particle, ligand-membrane and/or particle-membrane, repeatedly create feedback and influence each other. Ligand exchange occurring at the nano-cell membrane interface will inevitably lead to some unique interface phenomena and a variety of dynamic outcomes. However, there is currently a general lack of understanding of relevant interfacial ligand exchange mechanisms. The present study examines the effects of NP ligands and ligand exchange on the nano-bio interface with the goal of establishing general principles applicable to the rational design of bioactive NPs.

Here we have designed a cohort of model nanomaterials, gold nanoparticles (AuNPs) modified with 12 different kinds of ligands, to probe the roles of exchangeable ligands (exchanging with phospholipids) at the nano-membrane interface. We first utilized the quartz crystal microbalance with dissipation (QCM-D) technique to acquire kinetic and thermodynamic information 
about the ligand exchange in the nano-membrane interface interactions. Subsequently, we developed a combined imaging method, integrating AFM, ESEM and TEM, to comprehensively observe the morphology and ultrastructure of the nano-membrane interface, using both a nanosupported lipid bilayer (SLB) mimic and natural cell membranes. With the assistance of molecular dynamics (MD) simulations, we experimentally illustrate how exchangeable ligands dominate characteristic nano-bio interface phenomena and influence cell membrane integrity, uptake efficiency, and endocytosis of NPs.

\section{RESULTS}

Characteristics of NPs and membrane mimics. We synthesized Cit-AuNPs(-) by the citrate reduction of $\mathrm{HAuCl}_{4}{ }^{25}$ with a physisorption pattern and negative surface charges. To systematically explore the roles of ligands in the nano-membrane interface interactions, we then prepared a family of AuNPs of the same core size ( $13 \mathrm{~nm})$ with different surface ligands (Table 1; details of the synthesis are provided in the Materials and Methods section). The ligands were categorized according to molecular weight, charge and bonding modes ('physisorption' or 'chemisorption'). The relevant characteristics are provided in Figures S1 and S2 and Table S1. Furthermore, we constructed three types of SLBs with different charges (the structural formulas are provided in Figure S3): the neutral POPC, negatively charged DOPC/DOPS(-) and positively charged DOPC/DOEPC(+), all supported on silicon dioxide-coated, piezoelectric crystals in a QCM-D device (Figure S4). The processes and kinetics of the interactions between SLBs and AuNPs coated with different ligands were monitored by measuring the frequency variation $(\Delta F)$, which can be used to evaluate the mass of NPs adsorbed onto the SLBs, which is the reflection of the interaction intensity (Figure S5).

Roles of physisorbed ligands in nano-membrane interactions. As revealed by the " $\star$ " symbols (strong interactions) in Table 1, regardless of the ligand on the AuNP surface, the elec- 
trostatic interaction appears to be a universal driving force for NP adsorption onto charged lipid bilayers. Smaller molecule ligands could not fully shield the particle surface energy or high reactivity, leading to weak interactions (denoted by the "㝋" symbol) between AuNP-S- $\mathrm{C}_{11} \mathrm{OOH}(-$ ) or AuNP-S-C ${ }_{11} \mathrm{ONHC}_{2} \mathrm{NH}_{2}(+)$ and SLBs with like charges (Table 1 and Figure S5a, b). When PEG polymers were used to modify AuNPs (AuNP-S-PEG-COOH(-), AuNP-S-PEG-NH ${ }_{2}(+)$, AuNP-S-PEG-OCH 3 ), the electrostatic interaction became the only factor (Table 1 and Figure S5c-e), protecting AuNPs against self-aggregation. The situation became more complicated when AuNP surfaces were modified with bio-macromolecules (ssDNAP36 and BSA), because of the involvement of more types of interaction forces, such as hydrophobic interactions, hydrogen bonds and locally charged patches, etc., which led to weak interactions of NPs with neutral or the same charged SLBs (Table 1 and Figure S5f, g).

Our results demonstrate that all AuNPs covered with bonded ligands were either not absorbed at all or only weakly adsorbed onto neutral POPC bilayers, while AuNPs covered with unbonded ligands (e.g., Cit-AuNPs(-) or AuNPs with adsorbed short ssDNA) could interact intensely with the neutral bilayers (Table 1 and Figure S5h-j). Cit-AuNPs(-) are typically stabilized by adsorbed citrate ions, which provide just enough repulsion to prevent AuNPs from self-aggregation due to the strong van der Waals (VDW) attraction between AuNP particles. ${ }^{15}$ The affinity of citrate ions to AuNPs is relatively weak, considering it is only several $\mathrm{kJ} / \mathrm{mol}$ of desorption energy. ${ }^{26,27}$ In the case of DNA, it has been found that ssDNA can stick to AuNPs via DNA base adsorption, resulting in a more stable nano colloid system compared with citrate. ${ }^{28,29}$ The energy of desorption from AuNPs for a nucleoside base is about 100-120 kJ/mol, where G, C and A adsorb more strongly than T. ${ }^{30,31}$ Similarly, AuNPs modified with short ssDNA in the physisorption mode (AuNP@ $\mathrm{T}_{8}(-)$, AuNP@ $\left.\mathrm{T}_{18}(-)\right)$ interacted with neutral POPC bilayers, but to a lesser degree than Cit-AuNPs(-); this interaction shows a base-length dependence (Table 1, and Figure 
S5i, j). AuNPs modified with the same length ssDNA strands in the bonding mode (AuNP-S$\mathrm{T}_{8}(-)$, AuNP-S-T $\left.\mathrm{T}_{18}(-)\right)$ did not show a detectable interaction with the neutral and negative bilayers, but did so with positively-charged bilayers, driven by electrostatic interaction (Table 1 and Figure S5k, 1). These results reveal that the unbonded ligands play key roles in NP-bilayer interactions.

\section{Interfacial features involving physisorbed ligands. The Cit-AuNPs(-) represent AuNPs} with exchangeable small-molecule ligands with a negatively charged surface. The adsorption isotherm profile of the [Cit-AuNP+POPC] system resembled a "Type III" isotherm, ${ }^{32}$ resulting typically from a positive synergistic interaction (Figure S6). AFM imaging revealed the microstructures of the nano-bio interface of the Cit-AuNPs(-) interacting with the different membrane systems (Figure 1a-c). When Cit-AuNPs(-) $(100 \mu \mathrm{g} / \mathrm{mL})$ were incubated with the neutral POPC bilayers for $3 \mathrm{~h}$, agglomerated particles appeared on the SLBs (Figure 1b). High-resolution images show that the planform dimensions of the agglomerated particles reached into the hundreds of nanometers. Interestingly, the height of the agglomerate, however, still remained $\sim 13 \mathrm{~nm}$, indicating that AuNPs formed a monolayer aggregation on the neutral interface of POPC.

In the case of the negatively charged bilayer DOPC/DOPS(-), the Cit-AuNPs(-) also formed a monolayer aggregation, but at a lower scale, which could be attributed to the electrostatic repulsion (Figure 1a). When the Cit-AuNPs(-) were incubated with the positively charged bilayers DOPC/DOEPC (+), the electrostatic attraction facilitated the adsorption of Cit-AuNPs(-) onto the surface of the SLBs and resulted in aggregation on a larger scale and a multilayer accumulation (Figure 1c). When the ligand was changed to the polymer-ligand-modified AuNPs (AuNP-SPEG-COOH(-)), few NPs were found on the negative bilayer DOPC/DOPS(-) (Figure 1d), or the neutral membrane bilayer POPC (Figure 1e). Even when interacting with the positively 
charged membrane DOPC/DOEPC(+), the adsorbed dimension of AuNP-S-PEG-COOH(-) was much smaller than that of the [Cit-AuNP+DOPC/DOEPC] system (Figure 1f). High-resolution images display dispersed particles on the DOPC/DOEPC $(+)$ rather than aggregated ones. The adsorption isotherm (Figure S7) could be classified as a Langmuir-like model, that is typically a monolayer adsorption driven by electrostatic forces. ${ }^{32}$ Based on the above results, it can be inferred that the physisorbed ligands (citrate) dominate a particular interaction mode at the nanomembrane interface, during which electrostatic interactions can affect the degree of interaction, but not the nature of the interaction.

Insertion of Cit-AuNPs into synthetic lipid membrane micelles. We performed further experiments using vesicles in the place of the bilayers. A fluorescence quenching method based on a lipophilic dye was developed and the Cit-AuNP itself was used as a quencher (Figure 1g). Quantitative measurements of the fluorescence intensity showed that the degree of fluoresence quenching largely depends on the concentration of Cit-AuNPs(-) (Figure 1h). This suggests that the AuNPs were in close contact with the dye within a distance of around $10 \mathrm{~nm} .{ }^{33}$ Wang et al. found that fluid DOPC liposomes can promote the aggregation of Cit-AuNPs on the surface of liposomes, which may be attributed to the relatively faster diffusion within fluid bilayers and local lipid gelation. ${ }^{34}$ Herein, TEM examination showed that the AuNPs inserted themselves into the lipid membrane (Figure 1i). The fluidity of the lipid membrane makes it possible to embed particles with a larger size. Considering the hydrophobic surface of naked AuNPs, one of the most likely mechanisms for the interaction is a hydrophobic interaction between the naked AuNPs and hydrocarbon groups of the phospholipids, during which the citrate molecules are displaced by lipid molecules, while the AuNPs are inserted into the lipid layer.

Ligand exchange between NPs and lipid membranes. ATR-IR spectra of pure $\mathrm{Na}_{3} \mathrm{Cit}$ (Figure S8) shows two distinct peaks around 1500-1630 and 1305-1415 $\mathrm{cm}^{-1}$ which are 
respectively assigned to the asymmetric and symmetric stretching vibrations of the carboxylate group. ${ }^{15,35}$ Citrates on the surface of AuNPs include coordinated (coordination with the gold surface) and dangling citrate species, which connect through hydrogen bonding of carboxylic acid groups. ${ }^{15,27}$ The purified Cit-AuNPs were prepared by centrifuging repeatedly under basic conditions ( $\mathrm{pH}$-9) where the intermolecular interactions of $\mathrm{COOH}$ hydrogen bonds were interrupted. The resulting IR spectra homogeneously originate from citrate species coordinated directly with the AuNP surface. The vibrational frequencies of carboxylate groups rely heavily on their coordination modes, ${ }^{36}$ which lead to vibrational peak broadening of the adsorbed carboxylate groups on AuNPs (Figure 2a). However, these characteristic vibrational peaks of adsorbed carboxylate groups disappeared after interaction of Cit-AuNPs(-) with the POPC liposome solution (Figure 2a); the spectrum profile tended to be consistant with the pure POPC species (Figure S9), indicating the replacement of adsorbed citrates by the POPC species on the surface of AuNPs. Similarly, we employed XPS to further investigate the exchange of citrate ligands at the nano-membrane interface. The binding energy of the $\mathrm{C} 1 \mathrm{~s}$ and $\mathrm{O} 1 \mathrm{~s}$ from pure $\mathrm{Na}_{3} \mathrm{Cit}$, POPC species (Figure S10, S11), and $\mathrm{Na}_{3} \mathrm{Cit}$ adsorbed on AuNPs (Figure 2b, c) could be easily distinguished. The dominant peaks of the free or Au-coordinated carboxylate groups of Cit-AuNPs(-) as prepared (both $\mathrm{C} 1 \mathrm{~s}$ and $\mathrm{O}$ 1s) dispeared and changed into an XPS spectrum characteristic of POPC scpecies after intercation of Cit-AuNPs(-) with a POPC liposome solution, indicating the replacement of adsorbed citrates by POPC species on the surface of the AuNPs.

To better understand the process of ligand exchange in detail, we performed coarse-grained molecular dynamics simulations using the dissipative particle dynamics (DPD) technique. Typically, a citrate bilayer was constructed on AuNPs based on a trimeric unit consisting of two adsorbed and one dangling species which connected to each other through hydrogen bonding (Fig- 
ure S12). The coverage of adsorbed citrates per NP was 22\% (Figure 2d) or 46\% (Figure S13), as calculated from the well equilibrated Cit-AuNPs in water. When the Cit-AuNPs(-) encountered the phospholipid bilayer, at both levels of coverage, the Cit-AuNPs(-) were likely to enter the hydrophobic interior of the lipid bilayer, due to the hydrophobic interaction provided by the solvent-accessible hydrophobic Au beads. During this process, the citrates desorbed from the surface of AuNPs, decreasing the citrate coverage to $1.2 \%$ as the citrates preferentially remained in the water rather than the hydrophobic environment. This can be seen in the inset image at $\mathrm{t}=22000 \tau$ of Figure $2 \mathrm{~d}$. The AuNPs in the lipid bilayer are expected to undergo random motion, possibly interacting with each other during the diffusion. In contrast to Cit-AuNPs in water, without the long range electric repulsion provided by the adsorbed citrates, bare AuNPs will aggregate when encountering each other in the lipid bilayer due to the strong interaction between the Au beads (Figure $2 \mathrm{~d}$ image at $\mathrm{t}=50000 \tau$ [arrows]). The aggregated AuNPs are clearly visible in the images of lipid density and height inset into Figure $2 \mathrm{~d}$ at $\mathrm{t}=50000 \tau$. The above processes were recorded (Video S1) and the results are consistent with the AFM images (Figure 1b). However, in the bonded-ligand case, AuNP-S-PEG-COOH(-), the surface of which is $83 \%$ covered with bonded hydrophilic ligands (PEG-COOH) at a grafted density $0.441 / r_{\mathrm{c}}{ }^{2}$, AuNPs barely attached to the lipid bilayer and remained suspended in water due to the lack of any special adhesion force (Figure 2e).

Key factors affecting ligand exchange. In another case, it has been found that ssDNAphysisorbed AuNPs (AuNP@ $\left.\mathrm{T}_{8}(-)\right)$ can interact with neutral POPC bilayers (Figure S5i), but to a lesser degree than Cit-AuNPs(-) (Figure S5h). The corresponding AFM images (Figure 3a-c) reveal that the density and dimension of the particles in the AuNP@T8/SLB systems were similar to those in the Cit-AuNP/SLB systems (Figure 1a-c), indicating a similar interaction mechanism, but a weaker interaction strength. Additionally, when thiolated AuNP-S-T8(-), used as a 
control group, interacted with the different SLBs (Figure 3d-f), its behavior was similar to that of AuNP-S-PEG-COOH(-) (Figure 1d-f); widely dispersed particles were found on the DOPC/DOEPC (+) bilayers (Figure 3f), which is in agreement with the characteristics of an electrostatic force-mediated interaction. The statistical analysis of the diameters and heights of the above NPs at the nano-membrane interface are shown in Figure 4a, b, which indicates that a more stable colloid system is unfavorable for ligand exchange. To uncover the key factors affecting ligand exchange, we additionally investigated the ligand exchange events in a series of polyTn strand-stabilized AuNPs (Tn: T8, T12, T15, T18), using the corresponding thiolated strands as controls. The sequences and modifications of the oligonucleotides are listed in Table S2. It should be noted that using oligonucleotides as the model of an exchangeable ligand is quite complex. The adsorption behavior of poly-T strands onto the surface of AuNPs was first investigated using molecular dynamics (MD) simulations. Representative images of state trajectories of the four systems at $140 \mathrm{~ns}$ are inset in Figure 4c. Next, key indexes based on the thicknesses of the ligand layer (L), the number of bonded bases and the binding energy per ssDNA strand were determined by averaging frames over 100-140 ns (Figure 4c). As expected, the interactions between AuNP@Tn and the POPC bilayers were strand length-dependent (Figure 4d), i.e., the degree of interaction was proportional to the size and adsorption affinity of ligands.

Here we have shown that a ligand's size and adsorption affinity are two key factors affecting ligand exchange with lipid molecules at the nano-membrane interface. This conclusion is based on the following: 1) In the case of Cit-AuNPs, the small size and weak adsorption affinity of the citrate ions on the nano-surface were conducive to ligand exchange with a higher interaction strength (Figure S5h); 2) For AuNP@Tn, both ligand size and adsorption affinity improved with increasing ssDNA strand length, generating conditions less favorable for ligand exchange (Figure 4c). For AuNP-S-Tn (which possess a desorption enthapy corresponding to alkanethiols of 
about $\left.167 \mathrm{~kJ} \mathrm{~mol}^{-1}\right),{ }^{37}$ ssDNA strands can "tie" tightly to the surface of AuNPs with a high density, which markedly enhances the affinity and coverage, and thereby inhibits ligand exchange (Figure 4d); 3) For BSA-stabilized AuNPs, it had previously been found that BSA can be adsorbed onto the surface of AuNPs and form a rigid corona. Although the desorption energy of BSA from AuNPs was estimated to be only in the range of several tens of $\mathrm{kJ} / \mathrm{mol},{ }^{38,39}$ the large volume of BSA significantly improved the thickness of the ligand layer, preventing ligand exchange (Figure S5g). Thus, a simplified two-dimensional assessment system based on ligand size and adsorption affinity as two key factors may be created to estimate the possibility of ligand exchange at the nano-membrane interface (Figure 4e). To more comprehensively understand ligand exchange events, additional potential factors (such as the NP's chemical composition, ligand physicochemical properties, media conditions, etc.) should be also considered. A reasonable strategy would be to select as many key factors as possible to establish a multi-dimensional assessment system to predict ligand exchange.

Ligand-exchange interactions at the surface of cells. Finally, we investigated ligandexchange behavior using natural cell membranes. We chose AuNP@T18(-) as a representative NP to explore the role of exchangeable ligands at a human lung carcinoma cell line (A549) cell membrane interface. ESEM images of untreated A549 cells show intact cell membranes covered uniformly with long microvilli (Figure 5a). After exposure to AuNPs with various ligands for 6 h, the AuNPs can be found on the membrane surface in SEM mode (bright spots/red arrows). The ESEM images reveal that AuNP@T18(-) particles aggregated on the cell membrane, depending on their concentration (Figure 5b, c). At the same time, the number of microvilli decreased substantially around the aggregated-AuNPs, indicating an immediate response to the foreign stimu$\operatorname{lus}^{40}$. In contrast, AuNP-S-T18(-) and AuNP@BSA aggregated to a lesser extent on the cell surface, and the microvilli still were present, suggesting that thiol-bonding or a BSA corona im- 
proves the stability of AuNPs, preventing their aggregation at the nano-cell membrane interface, ultimately reducing membrane damage (Figure 5d, e).

The TEM images (Figure 5f-i) show that AuNPs modified with the various ligands mainly localized around microvilli of the cell membrane (Figure 5III, VIII, and X) and were transported to endo-/lysosomes when internalized (Figure 5VI, VII, IX and XI). Au@T18 also aggregated on the surface of membrane microvilli (Figure 5III, IV), exhibiting characteristics of an exchangeable ligand-dominated nano-cell membrane interface (Figure 5V). In contrast, AuNP-S-T18(-) particles were better dispersed around the microvilli (Figure 5VIII), indicating that bonding by the sulfhydryl group improves the stability of AuNPs and decreases their aggregation on the membrane. As a result, monodispersed NPs were found in lysosomes (Figure 5IX). Similarly, including the protein molecule (BSA) improved steric repulsion and compromised ligand exchange, resulting in AuNP@BSA(-) nanoparticles deposited around microvillar membranes with less aggregation. In another case, AuNP-S-PEG-COOH(-) particles were not found near the microvilli of the cell (Figure S14), implying that PEG molecules effectively decrease the contact of NPs with cells. The above results show that various ligands and their bonding modes influence the interaction of NPs with the natural cell membrane and subsequent cellular trafficking and distribution.

Effect of ligand exchange on the cellular internalization of NPs. We next examined the direct effects of NPs on endocytosis, which can be triggered by nano-cell membrane interactions, using various inhibitors of this complex cellular process. (Detailed information on the inhibitors used is listed in Table S4.) Interestingly, the endocytic pathways mediated by NPs' surface ligands were quite diverse and proceeded via multiple pathways, rather than being specifically mediated by a particular receptor (Figure S15). The endocytosis pathways of AuNP@T18(-) at a 
series of concentrations was investigated (Figure 6a). The clathrin- and caveolae/lipid raftmediated pathways decreased, while the macropinocytosis pathway was stimulated when A549 cells were incubated with the higher concentrations of AuNP@T18(-) particles. Macropinocytosis is a dynamin-independent process, which differs from the caveolae-dependent and clathrinmediated pathways, and involves a larger-scale internalization $(0.5-10 \mu \mathrm{m}) .{ }^{13,41}$ Ligand exchange-dominated nano-membrane interfaces readily result in the emergence of larger-scale particle aggregation at increased concentrations of AuNP@T18(-), which favors the macropinocytosis pathway.

We also evaluated the efficiency of AuNP uptake by A549 cells using the following equation: $(\mathrm{d} Q / \mathrm{d} t) /\left(N_{\text {cell }} C_{0}\right)$, where $Q$ is the mass of AuNPs internalized by cells, $N_{\text {cell }}$ is the number of cells incubated with AuNPs, and $C_{0}$ is the initial concentration of AuNPs $(\mu \mathrm{g} / \mathrm{ml})$ in the apical chamber of the cell culture system (See Materials and Methods in the supporting information). The uptake efficiency did not increase, but declined with increasing initial concentrations of AuNPs, indicating that larger particle aggregation was unfavorable for the internalization of NPs (Figure $6 b)$. Our results also indicate that the efficiency of endocytosis mediated by the higher energyconsuming macropinocytosis pathway was lower than those regulated via the caveolaedependent or clathrin-mediated pathways. A549 cells took up AuNP@T18(-) at a higher efficiency than any of the other bonding-ligand modified NPs (Figure 6b). A likely reason for this is that the aggregation of AuNP@T18(-) at the nano-cell interface resulted in an increased local concentration of particles near the surface of the cell membrane, which in turn promoted the internalization of the particles. Generally, ligand exchange occurring at the nano-cell membrane interface not only affects the endocytosis pathway of particles, but also impacts uptake efficiency. 
Discussion and conclusions. The nano-membrane interface comprises specific physicochemical interactions, and the related dynamic outcomes between the nano-surface and membrane components. To explore the complicated process of such an interface, we must understand the forces governing these interactions. ${ }^{22-24,42}$ The DLVO theory under classical colloidal conditions is partly applicable to describe the interaction between nanoparticles and lipid bilayers (sphereon-flat geometry system) using the appropriate equations. ${ }^{22,43}$ DLVO theory breaks down within separations $<2 \mathrm{~nm}$, where the situation is further complicated with the appearance of other forces, including thermal fluctuations, steric forces and solvent interactions etc. ${ }^{22,44,45}$ At the nanomembrane interface, these long-range or short-range forces often intertwine or interact simultaneously or follow one another in some systematic order in space and/or time (Figure S16a, b). In our case, the hydrophobic interaction/force plays a very important role. This feature consists of the hydrophobic surface of AuNPs and the hydrocarbon groups of phospholipids. It has been proven that the hydrophobic interactions between both small molecules and macroscopic particles or surfaces is of surprisingly long range and much stronger than VDW attraction. ${ }^{22}$ For ligand-coated AuNPs, the hydrophobic surface of naked AuNPs is masked by the hydrophilic coatings from the aqueous phase, which interfere with hydrophobic interactions. However, the masking is incomplete and depends on the size and modified density of ligands. In the case of the SLBs, hydrocarbon groups are, in principle, shielded efficiently by the hydrophilic headgroups from the aqueous phase in the unstressed state. However, a more accurate description is that the hydrocarbon groups are always partially exposed to the aqueous phase, as long as there exists a stretching force or tensile stress, permitting a hydrophobic interaction to take place. By changing the mean hydrophilic headgroup area by only a few percent, a remarkable enhancement of the hydrophobic attraction can be obtained. ${ }^{46}$ 
The exchange of ligands occurring at the nano-membrane interface is a result of multiple force interactions. The incompletely-shielded hydrophobic force and attractive VDW force, as longrange forces, are the main attractive forces dominating the adsorption of AuNPs to the lipid bilayer surface from a long separation distance up to a very close position. At a critical proximity $(\mathrm{D} \rightarrow 0)$, the emergence of other, non-DLVO forces usually makes the net repulsive forces reach a maximum. To move closer to the phospholipid surface, or even embed into the lipid bilayers, NPs need to overcome these resistances, requiring that the hydrophobic forces be greater than the repulsive forces. Considering that the hydrophobic force is exponentially proportional to the separation distance between a hydrophobic pair-potential, it can be inferred that the thickness of the ligand layer (or the size of the ligand molecule) is a key factor in ligand exchange. Simultaneously, for ligand exchange, NPs need to extricate themselves from the adhesion of their ligand molecules to ultimately expose their primal hydrophobic surfaces. Undoubtedly, a relatively strong affinity of ligand molecules for the NP surface is disadvantageous for ligand exchange. However, it is difficult to quantitatively assess the effects of ligand affinity, since a considerable portion of the attraction is counteracted by the forces that arise at the nano-SLB interface.

The spherical morphology of NPs makes the direction of ligand affinity shift away from the direction of the pair potential between the ligand and lipid molecule by the angle " $\theta$ " (Figure S16a), resulting in a "pulling" effect on the ligand molecules (proportional to the $\theta$ angle), which impairs the ligands affinity for the nano-surface. As shown in Figure S17 and S18, a fraction of the ligand molecules (citrate or T8 strand) has been separated from the surface of the NP, sequentially along the spherical nano-surface from the outside edge to the bottom, as the NP (CitAuNPs or AuNP@T8) approaches the phospholipid bilayer. Needless to say, it is impossible to describe with certainty all the forces and correlatively shaped interactions at the nano-SLB interface. For real nano-cell membrane interactions, the situation becomes more complicated and un- 
predictable, due to membrane fluidity, heterogeneity of the cell surface and the involvement of additional components (ligand-receptor); it is expected that different cell types will have unique features that may add nuances to their interactions with nanomaterials. ${ }^{47,48}$ While it is impossible to account for every factor involved, a tractable approach is to elucidate key factors affecting interfacial interactions under experimental conditions and to implement conditional predictions theoretically. In the current work, we built a ligand exchange assessment system based on ligand size and adsorption affinity as the key factors feeding into a conceptual strategy to guide relevant explorations at the nano-bio interface.

In summary, our work serves as a first step toward understanding the nano-cell membrane interface from the point of view of ligand-exchange. Our results show that exchangeable ligandstabilized NPs can aggregate synergistically, side-by-side into lipid bilayers, driven by ligandexchange, based on ligand properties, namely size and adsorption affinity. Subsequently, these characteristic interface interactions can influence NPs' endocytic pathways, uptake efficiency, and effects on cell membrane integrity. Uncovering the roles of ligand exchange at the nano-cell membrane interface improves our understanding of the underlying mechanisms involved in the complicated nano-bio interface and provides guidance for the rational design of nanomaterials for biomedical applications.

\section{ASSOCIATED CONTENT}

\section{Supporting Information}

Detailed explanation of materials and methods, additional supplementary figures, and supplementary videos are included. This material is available free of charge via the Internet at.

\section{AUTHOR INFORMATION}




\title{
Corresponding Author
}

* Email: chenchy@ nanoctr.cn

\begin{abstract}
Author Contributions
X.Y. Wang and C.Y. Chen designed research; X.Y. Wang, X.F. Wang, X. Bai, L. Yan, T. Liu, and M.Z. Wang performed research; Y.T. Song, Z.J. Gu, and Q. Miao contributed new reagents/analytic tools; X.Y. Wang, X.F. Wang, G.Q. Hu, and C.Y. Chen analyzed data; X.Y. Wang and C.Y. Chen wrote the paper.
\end{abstract}

\section{Notes}

The authors declare no competing financial interest.

\section{ACKNOWLEDGMENTS}

This work was financially supported by the National Basic Research Program of China from the Ministry of Science and Technology (2016YFA0201600 and 2016YFE0133100), the National Natural Science Foundation of China (91543125, 31571025, 21320102003 and 11435002), Innovative Research Groups of the National Natural Science Foundation of China (11621505) and the National Science Fund for Distinguished Young Scholars (11425520).

\section{REFERENCES}

(1) Sharifi, S.; Behzadi, S.; Laurent, S.; Forrest, M. L.; Stroeve, P.; Mahmoudi, M. Chem. Soc. Rev. 2012, 41, 2323-2343.

(2) Nel, A.; Xia, T.; Madler, L.; Li, N. Science 2006, 311, 622-627.

(3) Nel, A. E.; Madler, L.; Velegol, D.; Xia, T.; Hoek, E. M.; Somasundaran, P.; Klaessig, F.; Castranova, V.; Thompson, M. Nat. Mater. 2009, 8, 543-557.

(4) Stark, W. J. Angew. Chem. Int. Edit. 2011, 50, 1242-1258. 
(5) Lin, J.; Zhang, H.; Chen, Z.; Zheng, Y. Acs Nano 2010, 4, 5421-5429.

(6) Lesniak, A.; Salvati, A.; Santos-Martinez, M. J.; Radomski, M. W.; Dawson, K. A.; Aberg, C. J. Am. Chem. Soc. 2013, 135, 1438-1444.

(7) Li, J.; Mao, H.; Kawazoe, N.; Chen, G. Biomater. Sci-UK 2017, 5, 173-189.

(8) Verma, A.; Uzun, O.; Hu, Y. H.; Hu, Y.; Han, H. S.; Watson, N.; Chen, S. L.; Irvine, D. J.; Stellacci, F. Nat. Mater. 2008, 7, 588-595.

(9) Verma, A.; Stellacci, F. Small 2010, 6, 12-21.

(10) Ding, H. m.; Ma, Y. q. Small 2015, 11, 1055-1071.

(11) Canton, I.; Battaglia, G. Chem. Soc. Rev. 2012, 41, 2718-2739.

(12) Beddoes, C. M.; Case, C. P.; Briscoe, W. H. Adv. Colloid Interface Sci. 2015, 218, 48-68.

(13) Zhu, M.; Nie, G.; Meng, H.; Xia, T.; Nel, A.; Zhao, Y. Acc. Chem. Res. 2013, 46, 622-631.

(14) Nam, J.; Won, N.; Bang, J.; Jin, H.; Park, J.; Jung, S.; Jung, S.; Park, Y.; Kim, S. Adv. Drug Del. Rev. 2013, 65, 622-648.

(15) Park, J. W.; Shumaker-Parry, J. S. J. Am. Chem. Soc. 2014, 136, 1907-1921.

(16) Salvati, A.; Pitek, A. S.; Monopoli, M. P.; Prapainop, K.; Bombelli, F. B.; Hristov, D. R.; Kelly, P. M.; Aberg, C.; Mahon, E.; Dawson, K. A. Nat. Nanotechnol. 2013, 8, 137-143.

(17) Zhao, F.; Meng, H.; Yan, L.; Wang, B.; Zhao, Y. Sci. Bull. 2015, 60, 3-20.

(18) Tenzer, S.; Docter, D.; Kuharev, J.; Musyanovych, A.; Fetz, V.; Hecht, R.; Schlenk, F.; Fischer, D.; Kiouptsi, K.; Reinhardt, C.; Landfester, K.; Schild, H.; Maskos, M.; Knauer, S. K.; Stauber, R. H. Nat. Nanotechnol. 2013, 8, 772-81.

(19) Monopoli, M. P.; Aberg, C.; Salvati, A.; Dawson, K. A. Nat. Nanotechnol. 2012, 7, 779-786. (20) Fritzinger, B.; Moreels, I.; Lommens, P.; Koole, R.; Hens, Z.; Martins, J. C. J. Am. Chem. Soc. 2009, 131, 3024-3032.

(21) Cedervall, T.; Lynch, I.; Lindman, S.; Berggard, T.; Thulin, E.; Nilsson, H.; Dawson, K. A.; 
Linse, S. Proc. Natl. Acad. Sci. USA 2007, 104, 2050-2055.

(22) Israelachvili, J. N., Intermolecular and Surface Forces. 3rd ed.; Elsevier: Oxford, 2011.

(23) Min, Y.; Akbulut, M.; Kristiansen, K.; Golan, Y.; Israelachvili, J. Nat. Mater. 2008, 7, 527538.

(24) Bishop, K. J. M.; Wilmer, C. E.; Soh, S.; Grzybowski, B. A. Small 2009, 5, 1600-1630.

(25) Liu, J.; Lu, Y. Nat. Protoc. 2006, 1, 246-252.

(26) Feng, J.; Pandey, R. B.; Berry, R. J.; Farmer, B. L.; Naik, R. R.; Heinz, H. Soft Matter 2011, 7, 2113-2120.

(27) Al-Johani, H.; Abou-Hamad, E.; Jedidi, A.; Widdifield, C. M.; Viger-Gravel, J.; Sangaru, S. S.; Gajan, D.; Anjum, D. H.; Ould-Chikh, S.; Hedhili, M. N.; Gurinov, A.; Kelly, M. J.; El Eter, M.; Cavallo, L.; Emsley, L.; Basset, J. M. Nat. Chem. 2017, 9, 890-895.

(28) Li, H. X.; Rothberg, L. Proc. Natl. Acad. Sci. USA 2004, 101, 14036-14039.

(29) Zhang, X.; Servos, M. R.; Liu, J. W. Langmuir 2012, 28, 3896-3902.

(30) Ostblom, M.; Liedberg, B.; Demers, L. M.; Mirkin, C. A. J. Phys. Chem. B 2005, 109, $15150-15160$.

(31) Demers, L. M.; Ostblom, M.; Zhang, H.; Jang, N. H.; Liedberg, B.; Mirkin, C. A. J. Am. Chem. Soc. 2002, 124, 11248-11249.

(32) Adamson, A. W.; Gast, A. P., Physical Chemistry of Surfaces. 6th ed.; John Wiley \& Sons: New York, 1997.

(33) Acuna, G. P.; Bucher, M.; Stein, I. H.; Steinhauer, C.; Kuzyk, A.; Holzmeister, P.; Schreiber, R.; Moroz, A.; Stefani, F. D.; Liedl, T.; Simmel, F. C.; Tinnefeld, P. Acs Nano 2012, 6, 31893195.

(34) Wang, F.; Curry, D. E.; Liu, J. Langmuir 2015, 31, 13271-13274.

(35) Park, J. W.; Shumaker-Parry, J. S. ACS Nano 2015, 9, 1665-1682. 
(36) Park, J.-W.; Shumaker-Parry, J. S. J. Am. Chem. Soc. 2014, 136, 1907-1921.

(37) Nuzzo, R. G.; Dubois, L. H.; Allara, D. L. J. Am. Chem. Soc. 1990, 112, 558-569.

(38) Ramezani, F.; Rafii-Tabar, H. Mol. Biosyst. 2015, 11, 454-462.

(39) Boulos, S. P.; Davis, T. A.; Yang, J. A.; Lohse, S. E.; Alkilany, A. M.; Holland, L. A.; Murphy, C. J. Langmuir 2013, 29, 14984-14996.

(40) Lewinski, N.; Colvin, V.; Drezek, R. Small 2008, 4, 26-49.

(41) Doherty, G. J.; McMahon, H. T. Annu. Rev. Biochem. 2009, 78, 857-902.

(42) Wang, F.; Liu, J. Nanoscale 2015, 7, 15599-15604.

(43) Hoek, E. M. V.; Agarwal, G. K. J. Colloid Interf. Sci. 2006, 298, 50-58.

(44) Brant, J. A.; Childress, A. E. J. Membrane Sci. 2002, 203, 257-273.

(45) Barry, E.; Dogic, Z. Proc. Natl. Acad. Sci. USA 2010, 107, 10348-10353.

(46) Leckband, D.; Israelachvili, J. Q. Rev. Biophys. 2001, 34, 105-267.

(47) Canton, I.; Battaglia, G. Chem. Soc. Rev. 2012, 41, 2718-2739.

(48) Wang, J.; Zhang, L.; Peng, F.; Shi, X.; Leong, D. T. Chem. Mater. 2018, 30, 3759-3767. 
Table 1. Results of CM-D measurements of the interactions between different ligand-modified AuNPs and SLBs with different charges.

\begin{tabular}{|c|c|c|c|}
\hline \multirow[b]{2}{*}{ Ligands } & \multicolumn{3}{|c|}{ SLBs } \\
\hline & $\begin{array}{l}\text { POPC } \\
\text { (neutral) }\end{array}$ & $\begin{array}{l}\text { DOPC/DOPS(-) } \\
\text { (negatively charged) }\end{array}$ & $\begin{array}{l}\text { DOPC/DOEPC }(+) \\
\text { (positively charged) }\end{array}$ \\
\hline \multicolumn{4}{|l|}{$\begin{array}{l}\text { Small molecules (thiolated) } \\
\text { (M.W. 218.36; 260.44) }\end{array}$} \\
\hline AuNP-S- $\mathrm{C}_{11} \mathrm{OOH}(-)$ & & is & $\star$ \\
\hline AuNP-S-C ${ }_{11} \mathrm{ONHC}_{2} \mathrm{NH}_{2}(+)$ & & $\star$ & 访 \\
\hline \multicolumn{4}{|l|}{$\begin{array}{l}\text { Polymers (thiolated) } \\
\text { (M.W. 2000; 2000; 2000) }\end{array}$} \\
\hline AuNP-S-PEG-COOH(-) & & & $\star \star$ \\
\hline AuNP-S-PEG-NH ${ }_{2}(+)$ & & $\star \star$ & \\
\hline \multicolumn{4}{|l|}{ AuNP-S-PEG-OCH ${ }_{3}$} \\
\hline \multicolumn{4}{|l|}{$\begin{array}{l}\text { Biomacromolecules (thiolated) } \\
\text { (M.W. 10802; 66430) }\end{array}$} \\
\hline AuNP-S-P36(-) & & 为 & $\star \star$ \\
\hline AuNP@BSA(-) & 㶦 & 皮 & $\star$ \\
\hline \multicolumn{4}{|l|}{$\begin{array}{l}\text { Biomolecules (non-thiolated) } \\
\text { (M.W. 189.14; 2371.6; 5413.6) }\end{array}$} \\
\hline Cit-AuNPs(-) & $\star \star \star$ & $\star \star$ & $\star \star \star \star \star$ \\
\hline AuNP@T8(-) & $\star$ & 拧 & $\star \star \star$ \\
\hline AuNP@T18(-) & it & & $\star \star$ \\
\hline
\end{tabular}

Biomolecules (thiolated)

(M.W. 2403.7; 5445.7)

AuNP-S-T8(-)

AuNP-S-T18(-)

$" \star$ " denotes strong interactions $(\Delta F>30 \mathrm{~Hz})$ between NPs and SLBs. An additional $\star$ represents a $\Delta F$ increment of $100 \mathrm{~Hz}$. The number of $\star$ symbols is proportional to the mass of AuNPs adsorbed onto SLBs. The symbol "经" denotes a weak interaction with $5 \mathrm{~Hz}<\Delta F<30 \mathrm{~Hz}$. Sites with no symbol exhibited little or no interaction with $\Delta F<5 \mathrm{~Hz}$. 


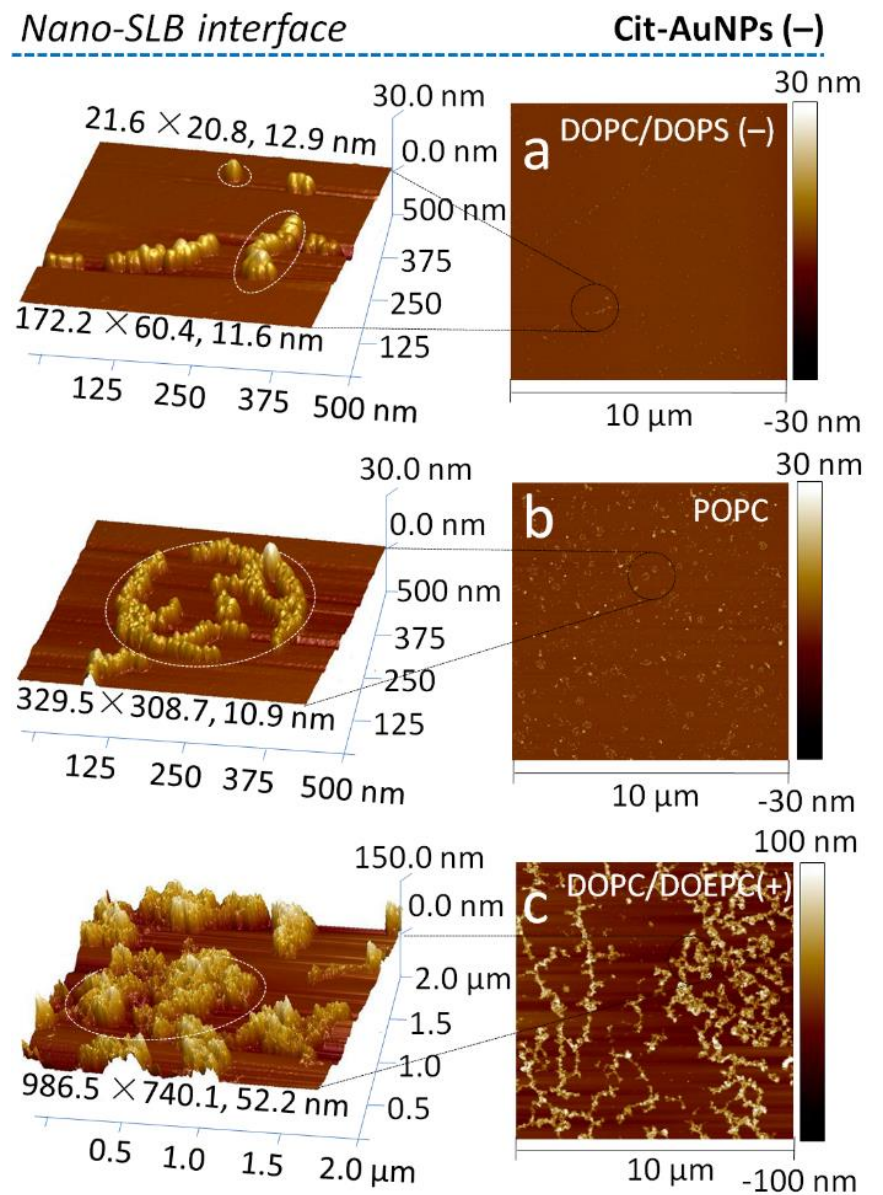

AuNP-S-PEG-COOH (-)

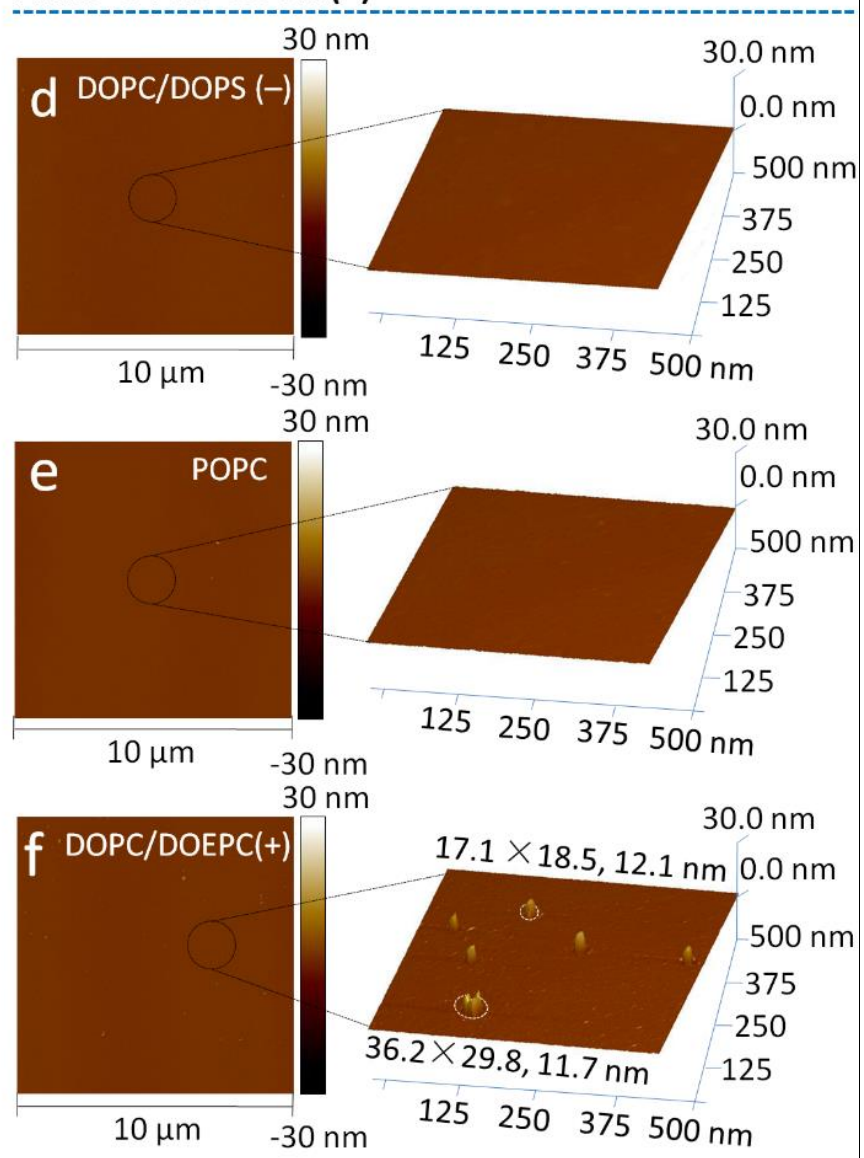

Nano-liposome interface
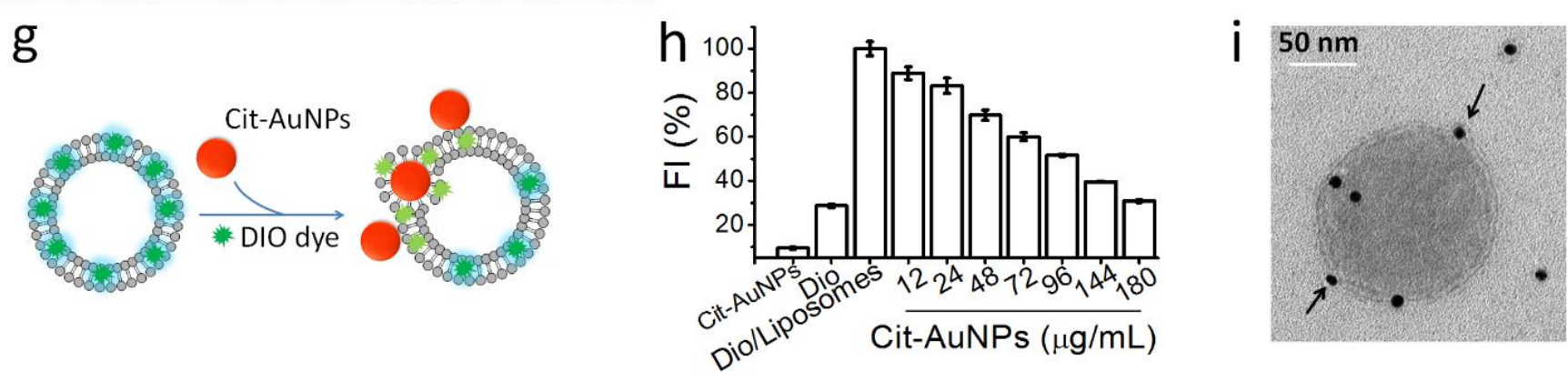

Figure 1. Interfacial features between Cit-AuNPs(-) and various lipid bilayers. $(a),(b)$ and $(c)$ AFM top view of the interaction of $100 \mu \mathrm{g} / \mathrm{mL}$ Cit-AuNPs(-) with DOPC/DOPS(-), POPC and DOPC/DOEPC(+) SLBs, respectively, with a $10 \mu \mathrm{m}$ scan size after $3 \mathrm{~h}$ of incubation. The images on the left are the regions encircled in black at a higher magnification with a scan size of 0.5 or $2.0 \mu \mathrm{m} .(d),(e)$ and $(f)$ AFM overview of the interaction of $100 \mu \mathrm{g} / \mathrm{mL}$ AuNP-S-PEG-COOH(-) with DOPC/DOPS(-), POPC and DOPC/DOEPC(+) SLBs, respectively, with a $10 \mu \mathrm{m}$ scan size after $3 \mathrm{~h}$ of incubation. The images on the right are the regions encircled in black at a higher magnification with a scan size of $0.5 \mu \mathrm{m} .(\mathrm{g})$ Schematic illustration of the interaction of CitAuNPs(-) with POPC liposomes based on fluorescence quenching. (h) Fluorescence quenching efficiency of Cit-AuNPs(-) with Dio dye-inserted POPC bilayers. The final concentration of vesicles was $2 \mathrm{mg} / \mathrm{mL}$, including $100 \mathrm{nM}$ of Dio dye ( $\lambda \mathrm{ex}=485 \mathrm{~nm}, \lambda \mathrm{em}=505 \mathrm{~nm}$ ), after mixing the same volume of Cit-AuNP solution from serial dilutions. (i) Representative TEM image of a POPC liposome incubated with Cit-AuNPs(-) (80,000x magnification). The sample was treated with osmium tetroxide solution $(2 \% \mathrm{w} / \mathrm{w})$. 


\section{Characterization of ligand exchange}
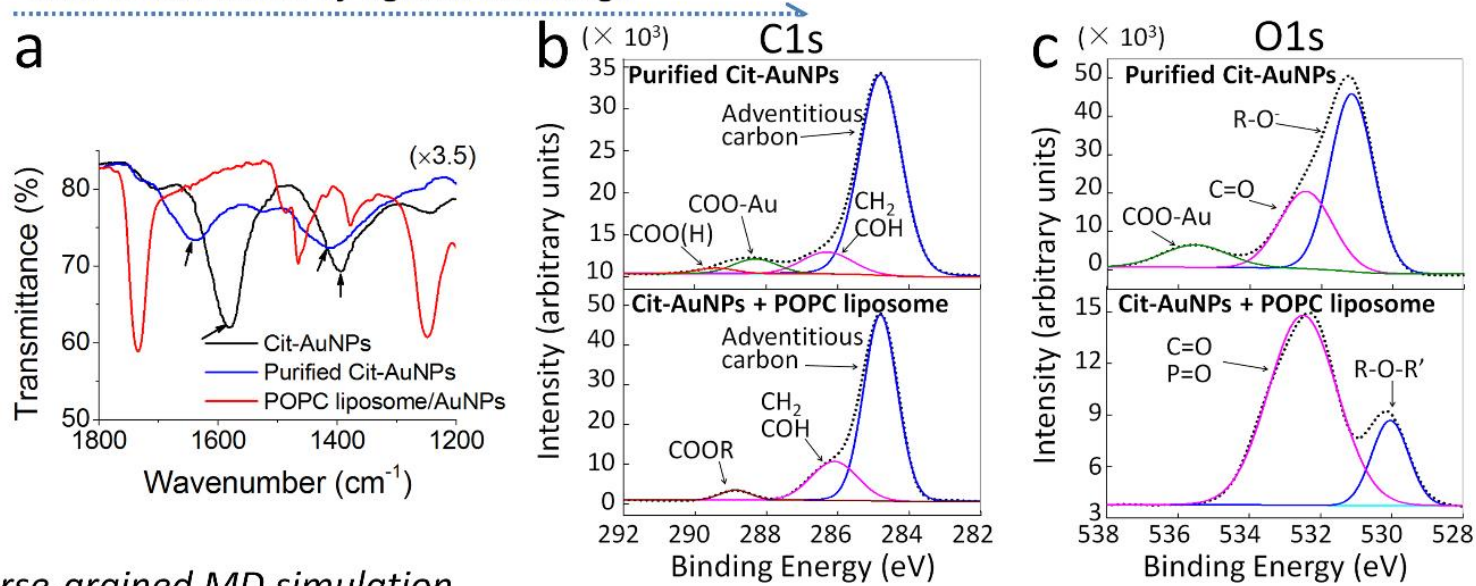

Coarse-grained MD simulation
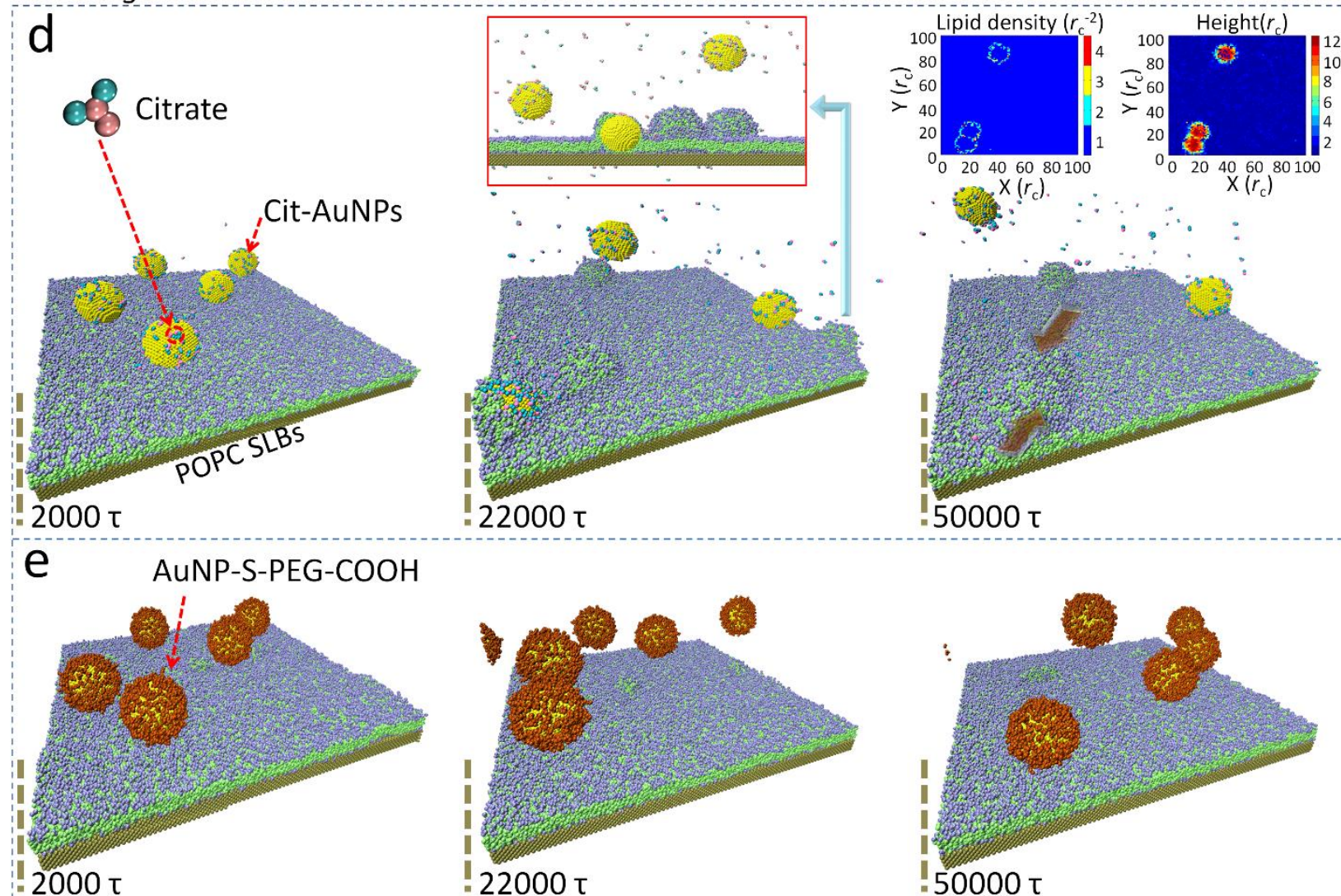

Figure 2. Ligand exchange occurring at the nano-membrane interface. (a) ATR-IR spectra of Cit-AuNPs, purified Cit-AuNPs and the NPs after interaction with an excess of POPC liposomes at the frequency region of $v_{\text {sy }}\left(\mathrm{COO}^{-}\right)$and $v_{\text {asy }}\left(\mathrm{COO}^{-}\right)$vibrations. The peaks of $v_{\text {sy }}\left(\mathrm{COO}^{-}\right)$at $1500-1600 \mathrm{~cm}^{-1}$ and $v_{\text {asy }}\left(\mathrm{COO}^{-}\right)$at $1400 \mathrm{~cm}^{-1}$ disappear upon the addition of the POPC liposomes (arrowed). (b) and (c) XPS spectra of the C1s and O1s binding energy of purified Cit-AuNPs and after interaction with an excess of POPC liposomes. (d) Coarsegrained molecular dynamics simulations using the DPD technique for time evolution of the interaction between the POPC SLBs and Cit-AuNPs(-). The inset image at $t=22000 \tau$ is the cross-section showing the insertion of AuNPs into the lipid bilayers. A lipid monolayer formed around AuNPs and the citrates were replaced during the interaction process. The inset images at $t=50000 \tau$ are top-view snapshots for the lipid density and height, coloured using the color scale bar on the right. (e) The coarse-grained molecular dynamics simulations using the DPD technique for time evolution of the interaction between the POPC SLBs and AuNP-S-PEG$\mathrm{COOH}$. 


\section{AuNP@T8(-)}

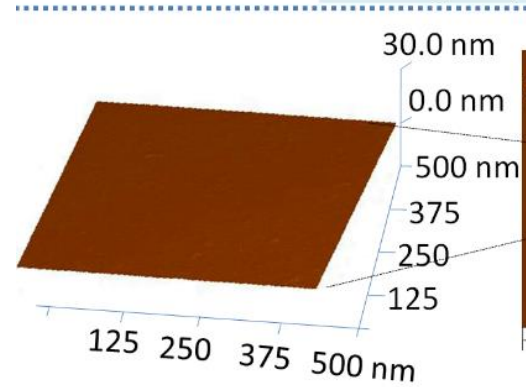

$30.0 \mathrm{~nm}$

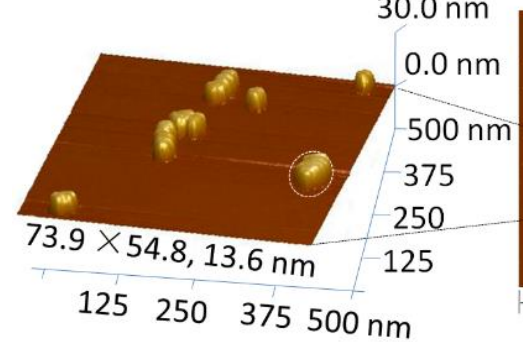

b
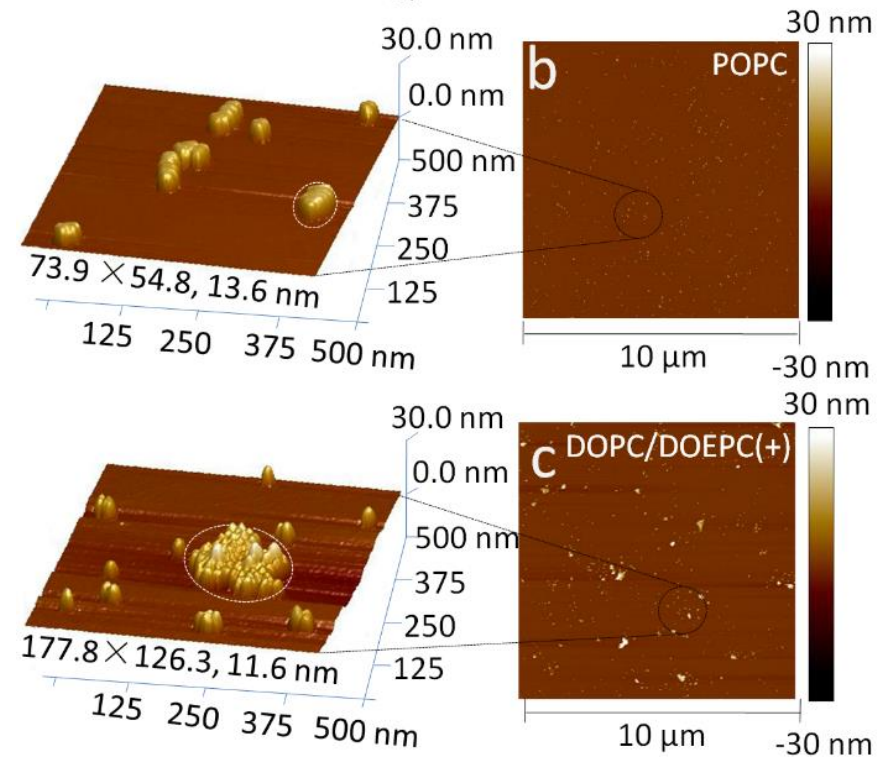

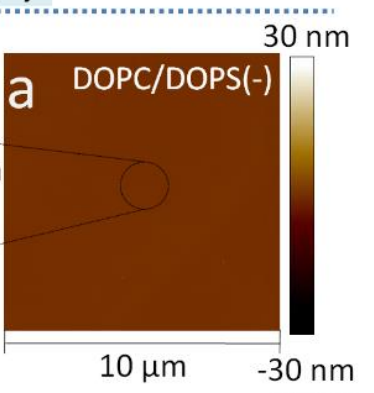

$30 \mathrm{~nm}$

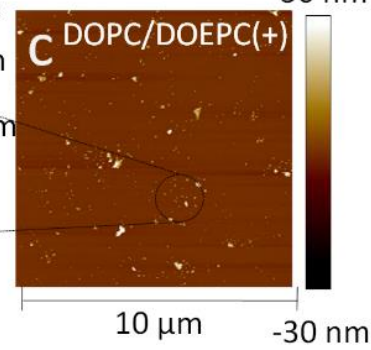

AuNP-S-T8(-)
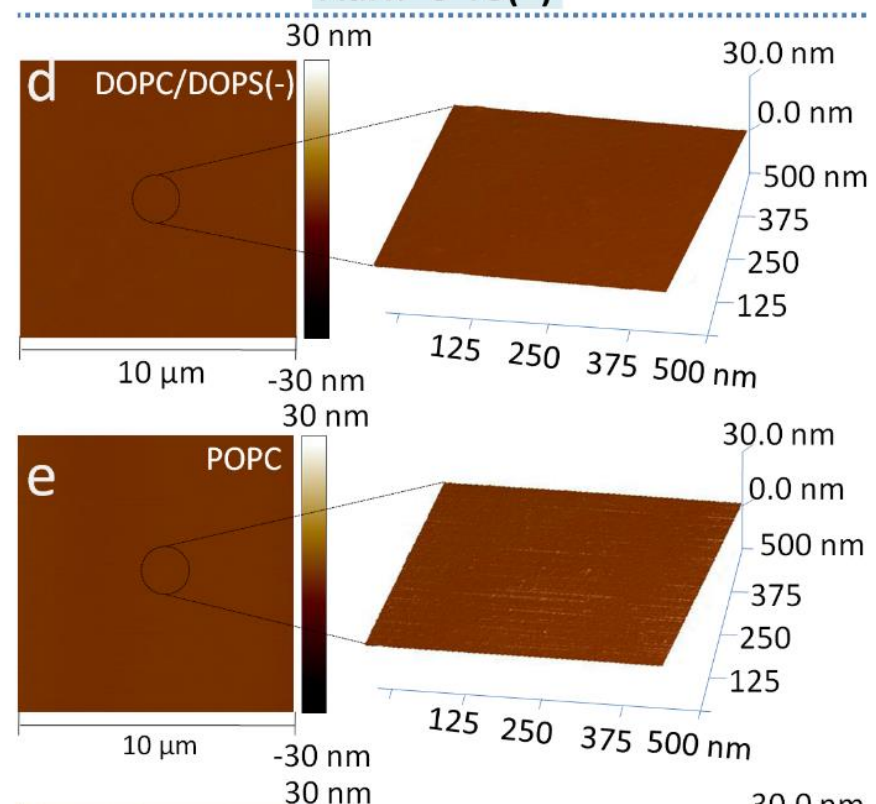

$125250 \quad 375500 \mathrm{~nm}$

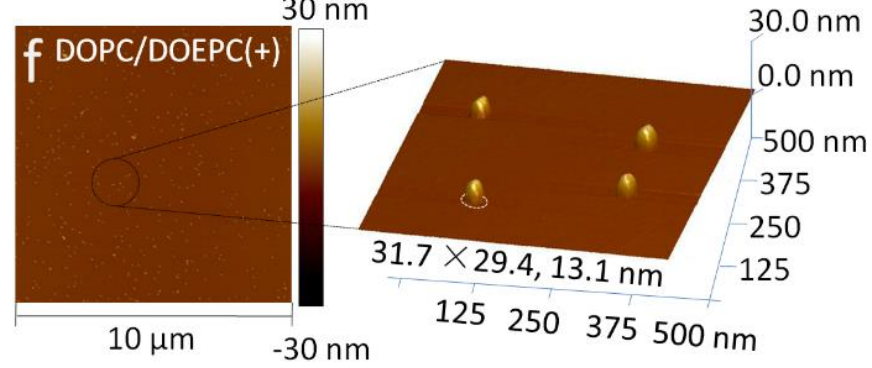

Figure 3. Microstuctures of oligonucleotide-adsorbed AuNPs at various lipid bilayer interfaces. $(a),(b)$ and $(c)$ AFM top view of the interaction of AuNP@T8(-) $(100 \mu \mathrm{g} / \mathrm{mL})$ with DOPC/DOPS(-), POPC and DOPC/DOEPC(+) bilayers, respectively, with a $10 \mu \mathrm{m}$ scan size after incubation for $3 \mathrm{~h}$. The images on the left correspond to the region in the black circle at a higher magnification with a scanning size of $500 \mathrm{~nm} .(d)$, $(e)$ and $(f)$ AFM top view images of the interaction of AuNP-S-T8(-) $(100 \mu \mathrm{g} / \mathrm{mL})$ with DOPC/DOPS $(-)$, POPC and DOPC/DOEPC (+) bilayers, respectively, with a $10 \mu \mathrm{m}$ scanning size after incubation for $3 \mathrm{~h}$. The images on the right correspond to the region in the black circle at a higher magnification with a scanning size of $500 \mathrm{~nm}$. 


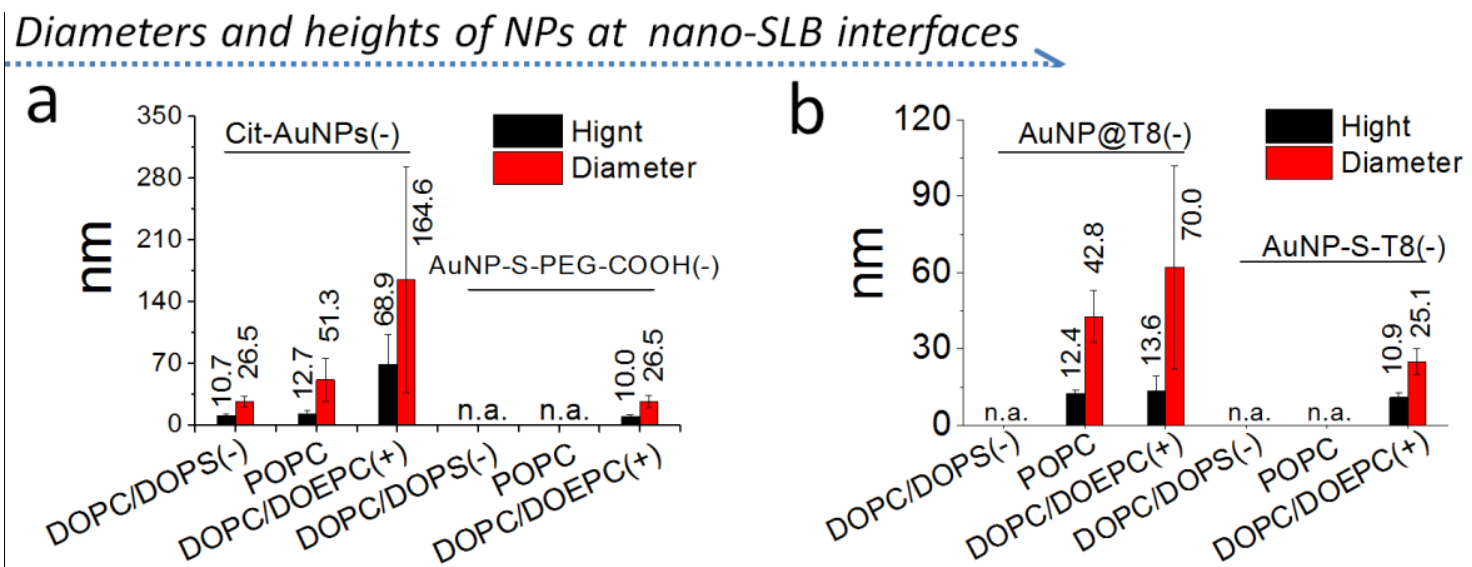

AuNP@Tn\&SLBs
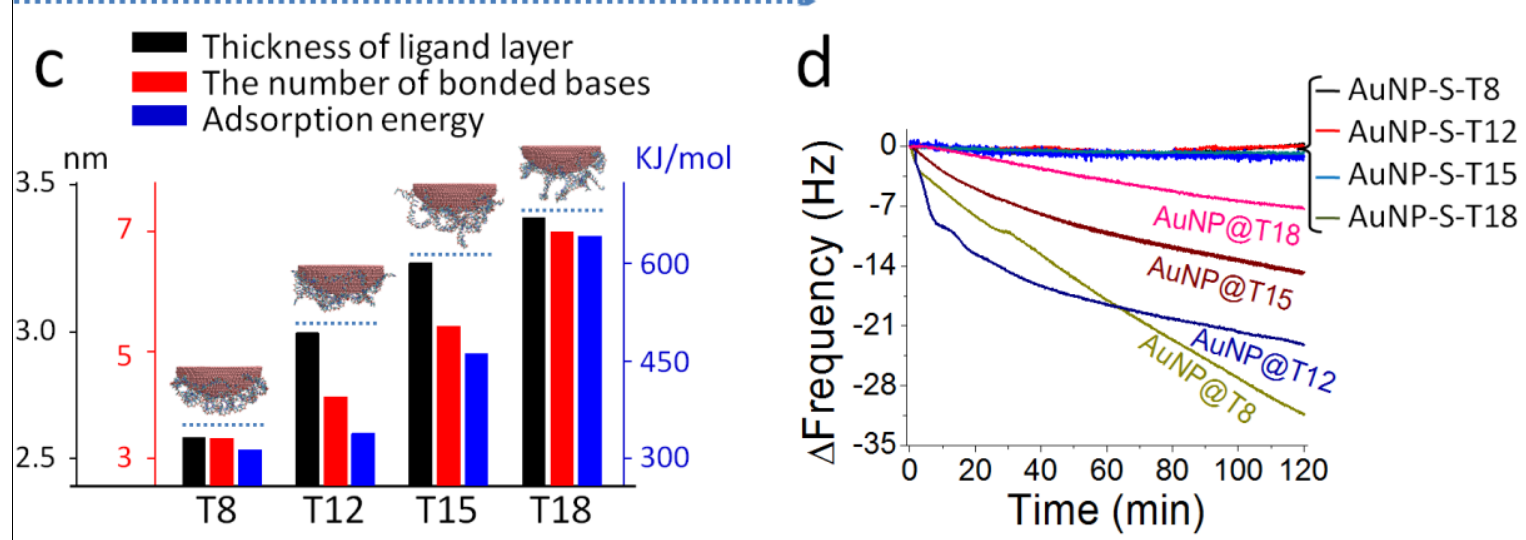

\section{Assessment of ligand exchange}

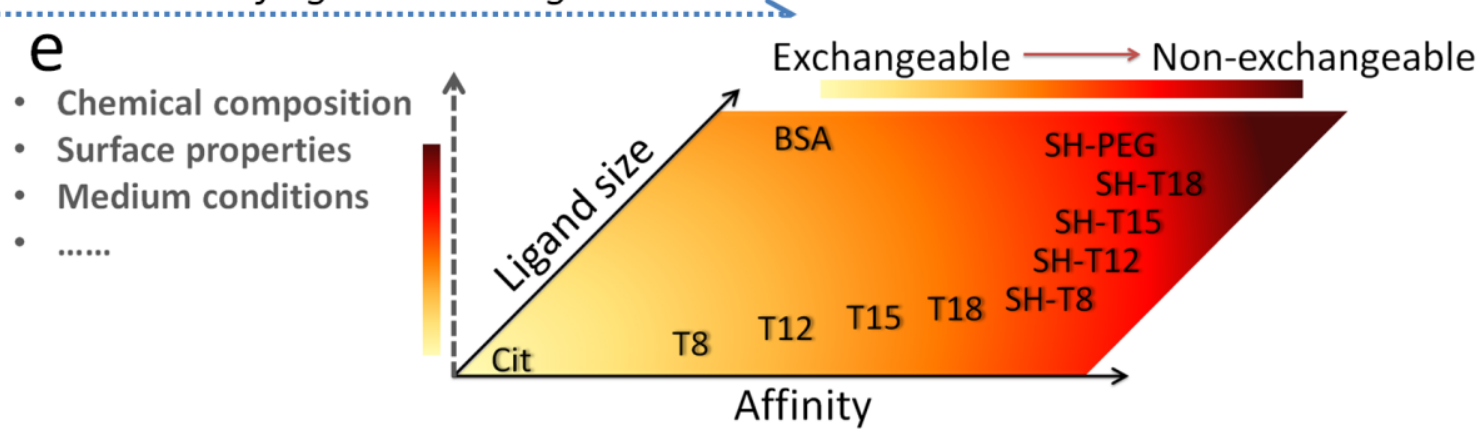

Figure 4. Key factors affecting the ligand exchange. $(a)$ and $(b)$ The statistical analysis of the diameters and heights of NPs after interacting with the indicated SLBs. The statistical results were obtained from more than 100 particles under $10 \mu \mathrm{m}$ scan size of imaging using NanoScope Analysis software. (c) The thickness of the ligand layer, the number of bonded bases and adsorption energy per poly-Tn strand on the surface of AuNPs based on MD simulation. Inserted images are representative snapshots from the state trajectories at $140 \mathrm{~ns} .(d)$ QCM-D monitoring of the adsorption kinetics of ssDNA-functional AuNPs onto POPC bilayers. (e) Assessing the possibility of ligand exchange on the surface of AuNPs, based on the affinity and size of ligand molecules. The color (from pale yellow to deep red) indicates the level of likelihood of ligand exchange. Other potential factors such as the nanoparticle's chemical composition, surface properties, and culture medium conditions etc., are suggested as additional dimensional vectors to establish a multi-dimensional assessment system. 


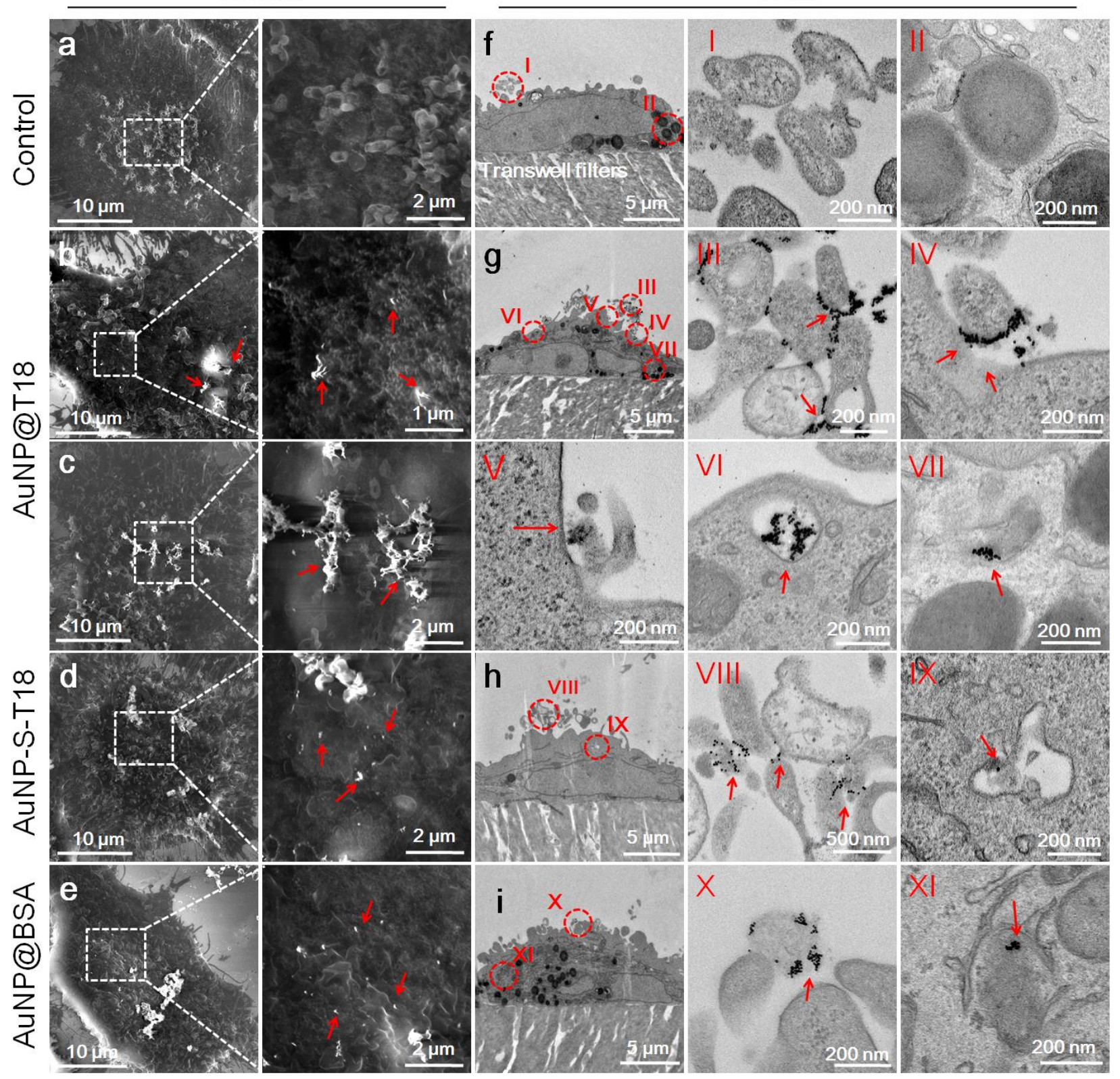

Figure 5. ESEM and TEM imaging of the nano-cell membrane interfaces and intracellular ultrastructures. The left-hand ESEM images are representative of whole, untreated A549 cells (a), A549 cells treated with 6 $\mu \mathrm{g} / \mathrm{mLAuNP} @ \mathrm{~T} 18(b), 18 \mu \mathrm{g} / \mathrm{mL}$ AuNP@T18 $(c), 18 \mu \mathrm{g} / \mathrm{mL}$ AuNP-S-T18 $(d)$ and $18 \mu \mathrm{g} / \mathrm{mL}$ AuNP@BSA $(e)$ for $6 \mathrm{~h}$. The right-hand ESEM images are magnification of the areas in the white dashed-line boxes in the corresponding images on the left. The TEM image in $(f)$ shows the cellular ultrastructures of untreated cells as controls. (I) and (II) are the magnification of microvilli and lysosomes, respectively, corresponding to the area in the red circle marked by red numbers in image f. The TEM image in $(g)$ shows the cellular ultrastructure after cells were exposed to $18 \mu \mathrm{g} / \mathrm{mL}$ AuNP@T18(-) for $6 \mathrm{~h}$. (III-VII) are the magnification of microvilli, the nano-cell membrane interface, macropinocytosis, an early endosome and lysosome, respectively, corresponding to the area of the red circle marked by red numbers in image $\mathrm{g}$ in the sequence. The TEM image in $(h)$ shows the cellular ultrastructure after cells were exposed to $18 \mu \mathrm{g} / \mathrm{mL}$ AuNP-S-T18(-) for $6 \mathrm{~h}$. (VIII) and (IX) are the magnification of the red dashed circle in image h showing microvilli and a lysosome, respectively. The TEM image in $(i)$ shows the cellular ultrastructures after cells were exposed to $18 \mu \mathrm{g} / \mathrm{mL}$ AuNP@BSA(-) for 6 h. $(\mathrm{X})$ and $(\mathrm{XI})$ are the magnification of the region in the red dashed circle in image I, showing microvilli and a lysosome, respectively. 

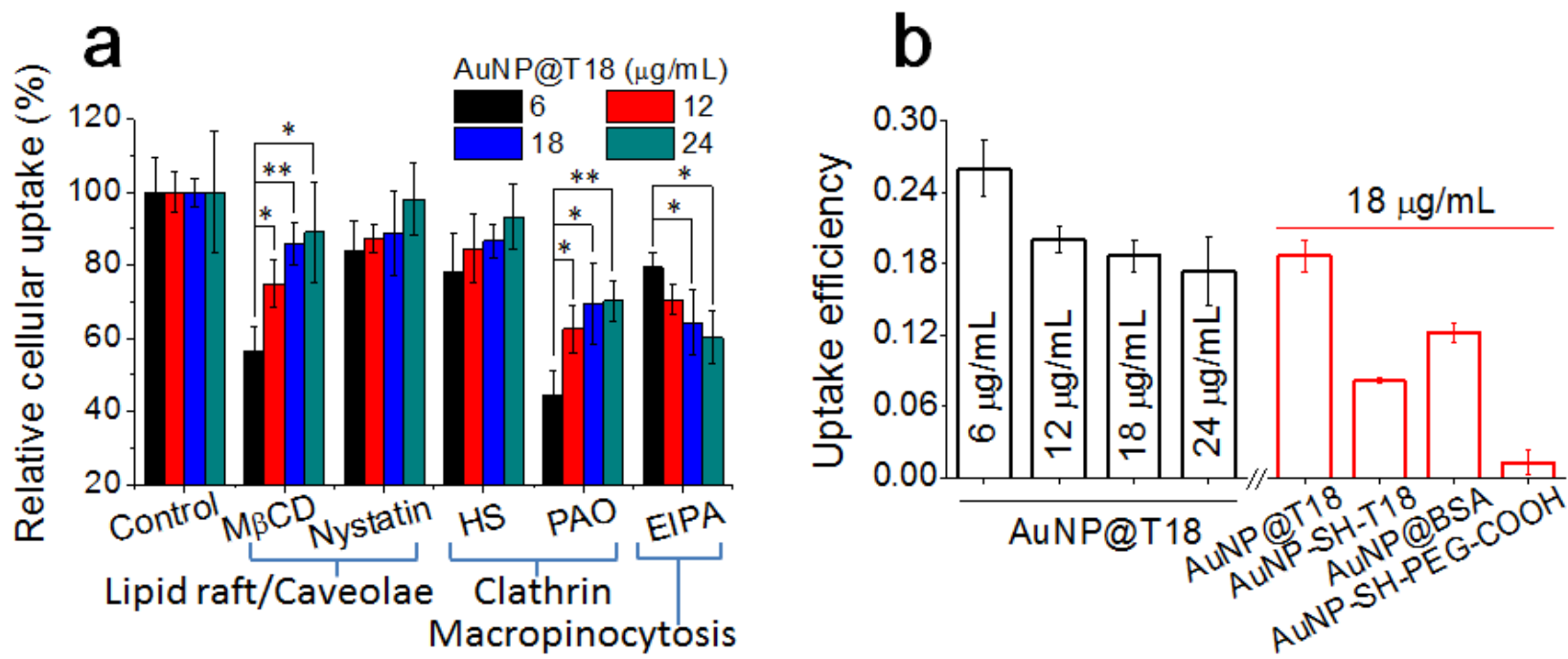

Figure 6. Effects of Ligand exchange behavior on the internalization of NPs by cells. (a) Endocytosis pathways of AuNP@T18(-) at the indicated concentrations in A549 cells, measured by ICP-MS. The incubation of cells with only AuNPs without inhibitors served as the control, set to $100 \%$. (b) Endocytosis efficiency of AuNPs with different ligands. The inhibitors and their concentrations used in uptake pathway experiments are listed in Table S4. All data are presented as the mean \pm SD of three replicates. $* P<0.05$ and $* * P<0.01$. 


\section{For Table of Contents Only}

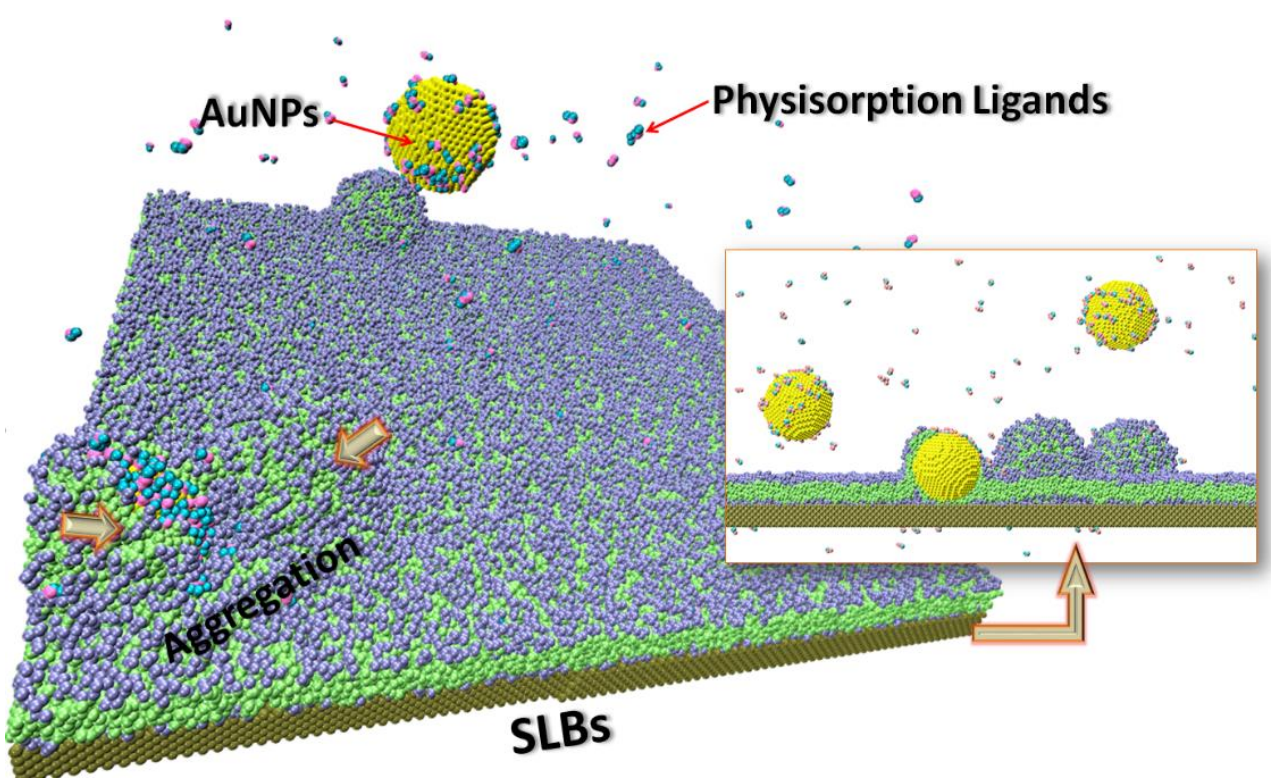

ACS Paragon Plus Environment 